\title{
Diagnosis of Bovine Sarcocystosis by Conventional Methods
}

\author{
Rasmita Panda*, Manchukonda Udaya Kumar, \\ G.S. Sreenivasa Murthy and Y.N. Reddy \\ Department of Veterinary Parasitology, College of Veterinary Science, PVNR TVU, \\ Rajendranagar, Hyderabad-500 030, Telangana, India \\ *Corresponding author
}

\section{Keywords}

Bovine, Sarcocystis, Bradyzoites, Staining

Article Info

Accepted:

10 January 2019

Available Online:

10 February 2019

\section{A B S T R A C T}

Sarcocystis species are considered as one of the most prevalent parasites of bovines. Sarcocysts occur either macroscopically or microscopically in the tissues of bovine intermediate hosts. Sarcocystosis being an occult infection, most of the time diagnosis in intermediate host by clinical symptoms is unreliable. In this study, the macrosarcocysts were observed in the infected muscle sample by naked eye examination. Suspected muscle samples were examined by muscle squash technique which showed the presence of microsarcocysts in the muscle tissue and bradyzoites were recovered through muscle digestion technique. The detailed morphology of the recovered bradyzoites was studied by Giemsa, Leishman's and Diff Quick staining which revealed banana shaped bradyzoites with a clear nucleus towards the posterior end. The trypan blue staining indicated live (colourless) and dead (blue) bradyzoites and the gliding movement of the bradyzoites was recorded.

\section{Introduction}

Sarcocystosis is characterised by cyst formation in muscle tissue (muscular sarcocyctosis) in the intermediate host or colonization of lamina propria of the intestines (intestinal Sarcocystosis) in definitive host. Sarcocystis has a requisite two-host life cycle based on a prey-predator (intermediate-definitive) host relationship (Fayer, 2004). The economic loss in livestock industry due to bovine sarcocystosis is enormous. This is mainly due to condemnation or down-grading of meat containing grossly visible sarcocysts (Fayer, 1976). Bovine sarcocystosis in India has been reported by many workers and the prevalence has been reported throughout the year. Diagnosis of acute sarcocystosis is difficult because the disease is generalised in nature with no specific signs. Conventional diagnostic methods such as muscle squash (Mowafy et al., 1993; Odening et al., 1996; Motamedi et al., 2010), muscle digestion (Collins et al., 1980; Hamidinejat et al., 2010; Mangas et al., 2015), histopathological examination (Ghisleni et al., 2006; Pritt et al., 2008) are available for postmortem diagnosis of bovine sarcocystosis.

In the present study various conventional methods were tried to recover the bradyzoites 
from the infected animals and then the bradyzoites were subjected to different staining techniques for identification.

\section{Materials and Methods}

Bovine oesophagi containing macrosarcocysts (Fig. 1) were collected from Chengicherla slaughter house, Hyderabad, Telangana, India and brought to the laboratory on ice. The cysts were gently dissected out and thoroughly washed in PBS ( $\mathrm{pH} 7.2)$. The size and shape of the various cysts collected were studied.

The muscle samples which did not show any cyst on gross examination were subjected to squash technique and acid pepsin digestion method. In squash technique, small pieces of muscle of about $5 \times 5 \times 2 \mathrm{~mm}$ were compressed between two glass slides and examined microscopically for Sarcocystis cysts as per the method described by Singh et al.(1990) and Dubey et al., (2000). In digestion technique, suspected muscles (20 g) were incubated at $40{ }^{0} \mathrm{C}$ for $20 \mathrm{~min}$ in $50 \mathrm{ml}$ of acid-pepsin (2.6 g pepsin, $5 \mathrm{~g}$ Nacl, $7 \mathrm{ml} 1 \mathrm{M}$ $\mathrm{Hcl}, 993 \mathrm{ml}$ distilled water).

This suspension was filtered through a $38 \mu \mathrm{m}$ sieve, centrifuged at $2000 \mathrm{rpm}$ for $5 \mathrm{~min}$, and sediment was suspended in $0.5 \mathrm{ml}$ of distilled water and a drop of this solution was examined for the presence of bradyzoites under light microscope as per the procedure laid down by Fischer and Odening (1998) and Barham et al., (2005).

The bradyzoites collected in digestion method were centrifuged and the sediment was washed thrice in PBS ( $\mathrm{pH}$ 7.2). Smears of bradyzoites were made on clean grease free glass slides and air dried. The dried smears were subjected to different types of staining such as Giemsa, Leishman's staining and Diff
Quick staining etc. Trypan blue staining was done to visualize the dead and live bradyzoites. For Diff Quick staining, the smear was dipped in the Diff Quick fixative (Absolute methanol) for five times. Then the slide was dipped 5 times each for one second in Eosin and the back of the slide was wiped off. This was followed by dipping the slide in methylene blue stain for 5 times each for one second. The back of the slide was wiped off, the smear was rinsed with distilled water and air dried. In Trypan blue staining, $10 \mu \mathrm{l}$ of bradyzoite suspension was mixed with $90 \mu \mathrm{l}$ of $0.4 \%$ Trypan blue stain $(0.4 \mathrm{~g}$ trypan blue in $100 \mathrm{ml}$ of PBS, $\mathrm{pH} 7.4$ ), mixed properly and one drop of the mixture was examined under microscope.

\section{Results and Discussion}

The sarcocysts collected were creamy white in colour, of different shapes viz. spindle, fusiform and globular etc. and of different sizes varying from $2.0-18.0 \mathrm{~mm} \times 1.0-5.0 \mathrm{~mm}$ (Fig. 2). In muscle squash method, micro sarcocysts in fusiform shape were found in the compressed muscle pieces (Fig. 3). In our experiment, the digestion of the muscle sample in acid-pepsin for twenty min was found to be equally sensitive as 1 hour digestion and bradyzoites (Fig. 4) were seen by examining one drop of the sediment of the digested muscle fluid. The stained smears of bradyzoites were focused under oil immersion lens.

The stained bradyzoites appeared in banana shape with clear and stained nucleus towards the posterior end (Fig. 5, 6, 7). In Trypan blue staining which was specifically used to differentiate the live and dead cells, the dead bradyzoites appeared blue in colour where as live bradyzoites did not take any stain (Fig. 8). 
Int.J.Curr.Microbiol.App.Sci (2019) 8(2): 941-947

Fig.1 Macro sarcocysts present on the oesophageal muscle

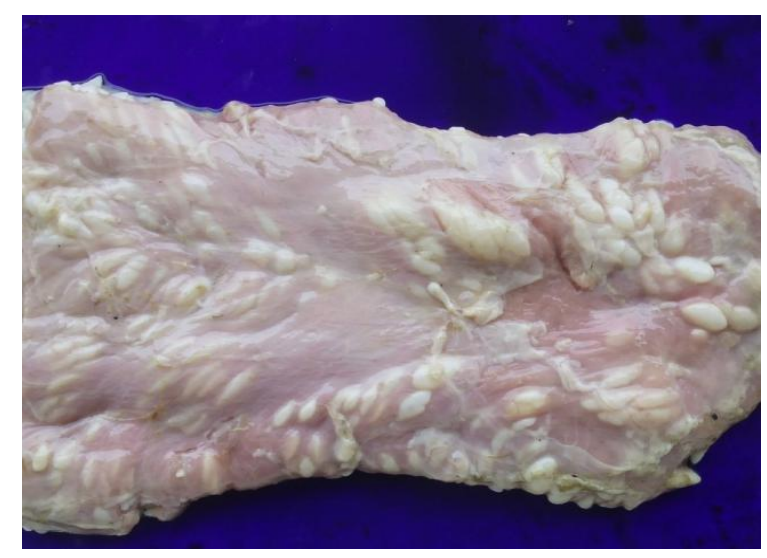

Fig.2 Macrosarcocysts of different shape and size

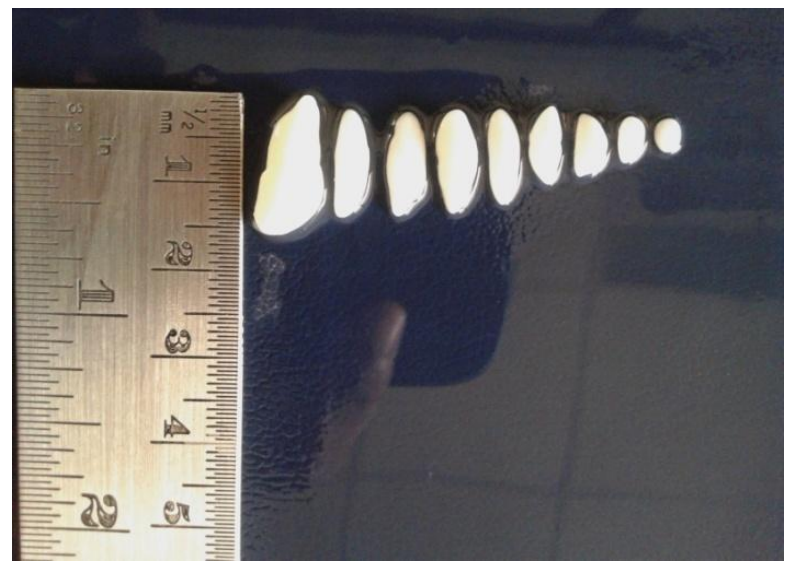

Fig.3 Microsarcocyst of Sarcocystis sp. obtained by muscle squash technique

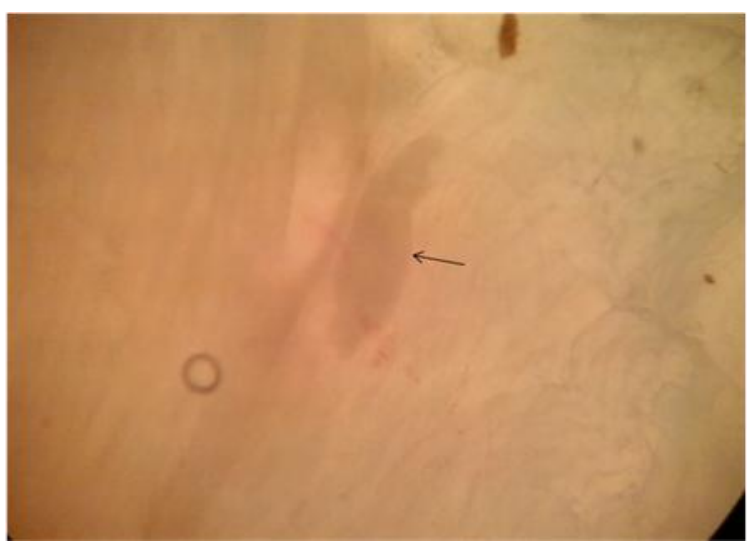


Fig.4 Bradyzoites of Sarcocystis sp. obtained by muscle digestion technique

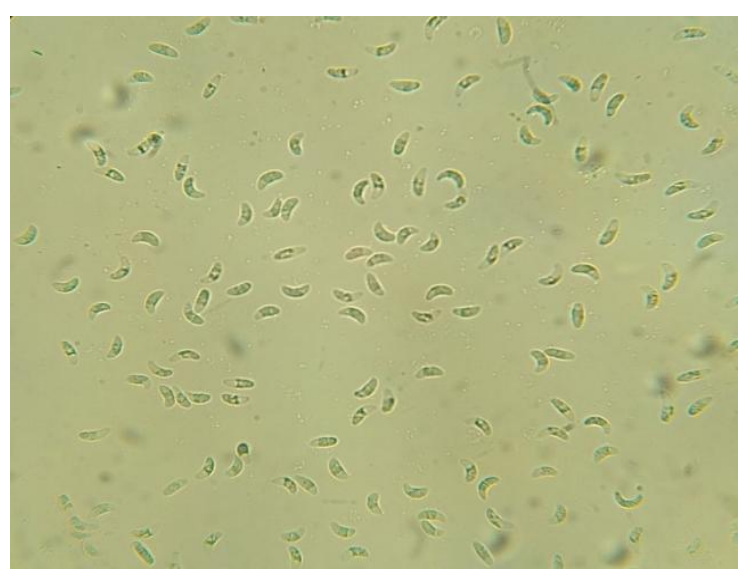

Fig.5 Giemsa stained bradyzoites

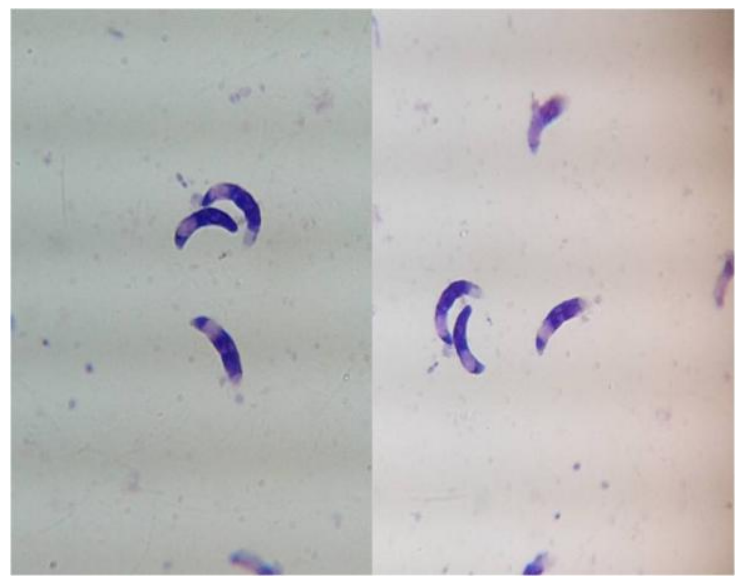

Fig.6 Leishman stained bradyzoites

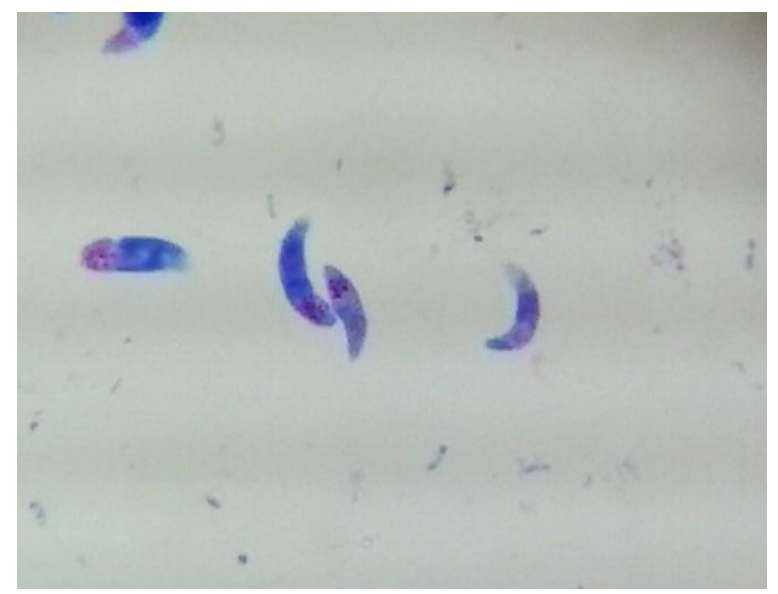


Fig.7 Diff Quick stained bradyzoites

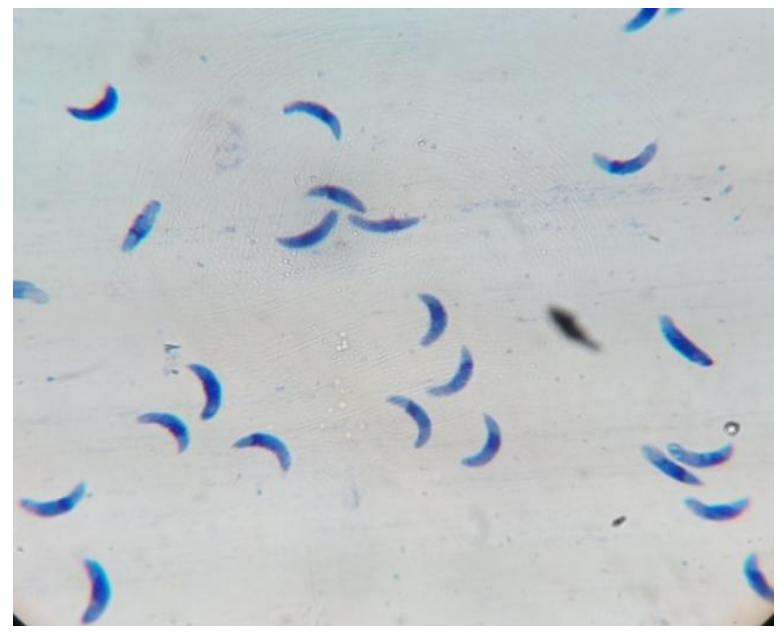

Fig.8 Live and dead bradyzoites

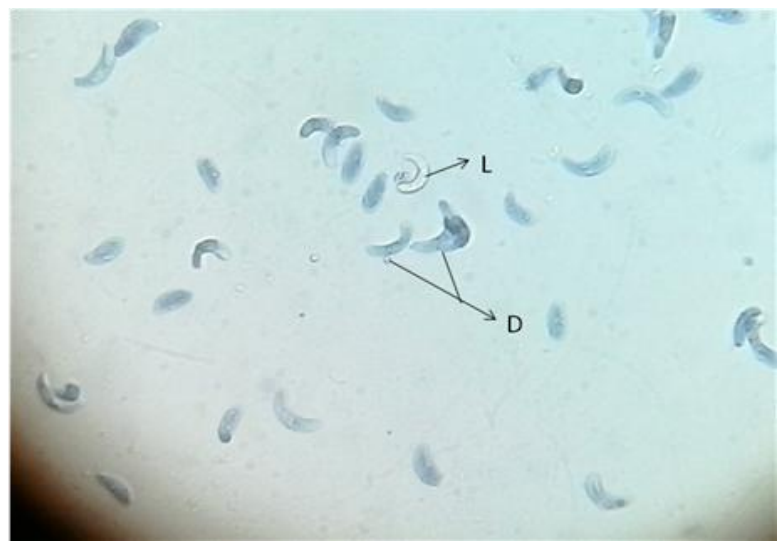

Sarcocystosis in bovine intermediate hosts can be confirmed during post-mortem examination only after demonstrating the macrosarcocysts in naked eye or by the detection of microsarcocysts in tissue samples. In our preliminary studies, the presence of bradyzoites in the esophageal muscles of bovines was ascertained by macroscopic, muscle squash and muscle digestion methods followed by Giemsa, Leishman's and Diff quick staining techniques.

On macroscopic observation, the macrosarcocysts $(2-18 \quad \mathrm{~mm} \quad \mathrm{x} \quad 1-5 \quad \mathrm{~mm})$ appeared in different shapes viz., globular, spindle, fusiform and like rice grain indicating the irregular expansion of the cyst depending up on the loose space available in various muscles and also the resistance by the host. Similar findings were recorded by Bhatia et al.,(2004) and Dasma Bai (2012). The cysts varied in size from a few micrometers to several centimeters, depending on the host and species (Merck, 2005).

The presence of microsarcocysts in the eosophagus could be demonstrated by muscle squash technique but the bradyzoites were not demonstrable by the same method which 
could be due to unruptured, intact cyst wall. Similar observations were made by Badawy et al., (2012). However the bradyzoites could be recovered by muscle digestion method described by Collins et al., (1980). In this study 20 min digestion of the muscle pieces by acid pepsin yielded good number of bradyzoites which was on similar lines with Collins et al., (1980) who suggested $20 \mathrm{~min}$ digestion as sensitive as 1 hour digestion.

The bradyzoites recovered by digestion method was washed thrice in PBS and subjected to different stains which revealed banana shaped bradyzoites with a nucleus at the posterior end by Giemsa and Leishman's staining techniques as reported earlier by Obijiaku (2012). A Diff quick staining technique described for staining merozoites of Toxoplasma gondii by Hehl et al., (2015) was applied for the staining of bradyzoites in our study and the stained bradyzoites showed a dark stained nucleus towards posterior end.

\section{Acknowledgment}

The first author is thankful to Indian Council of Agricultural Research and PVNR Telangana Veterinary University, Hyderabad, Telangana, India for providing necessary facilities to carry out the above research programme.

\section{References}

Badawy, A. I. I. 2013. Sarcocystis hominis and other Sarcocystis species infecting cattle at Sharkia Province, Egypt. J Am Sci. 8(8): 271-275.

Barham, M., Stützer, H., Karanis, P., Latif, B. M., and Neiss, W. F. 2005. Seasonal variation in Sarcocystis species infections in goats in northern Iraq. Parasitology. 130(02): 151-156.

Bhatia, B. B., Pathak, K.M.L. and Juyal, P. D. 2004 Textbook of Veterinary
Parasitology $\quad 3^{\text {rd }}$ edn Kalyani Publishers pp 497

Collins, G. H., Charleston, W. A. G., and Wiens, B. G. 1980. Studies on Sarcocystis species VI: A comparison of three methods for the detection of Sarcocystis species in muscle. New Zealand veterinary journal. 28(9): 173-173.

Dasma Bai, B. 2012. Diagnosis of bovine sarcocystosis by immunofluorescent antibody technique. M.V.Sc Thesis. Sri Venkatrswara Veterinary University, India.

Dubey, J. P., Saville, W. J. A., Lindsay, D. S., Stich, R. W., Stanek, J. F., Speer, C. A., Rosenthal, B.M., Njoku, C.J., Kwok, O.C.H., Shen, S.K. and Reed, S.M. 2000. Completion of life cycle of Sarcocystis neurona. Journal of Parasitology. 86: 1276-1280

Fayer, R. 2004. Sarcocystis spp. in human infections. Clinical Microbiology Reviews. 17: 894-902.

Fayer, R., Johnson, A. J., and Lunde, M. 1976. Abortion and other signs of disease in cows experimentally infected with Sarcocystis fusiformis from dogs. Journal of Infectious Diseases. 134(6): 624-628.

Ghisleni, G., Robba, S., Germani, O., and Scanziani, E. 2006. Identification and prevalence of Sarcocystis spp. cysts in bovine canned meat. Food Control., 17(9): 691-694.

Hamidinejat, H., Razi Jalali, M. H., and Nabavi, L. 2010. Survey on Sarcocystis infection in slaughtered cattle in south-west of Iran, emphasized on evaluation of muscle squash in comparison with digestion method. Journal of Animal and Veterinary Advances. 9(12): 17241726.

Hehl, A. B., Basso, W. U., Lippuner, C., Ramakrishnan, C., Okoniewski, M., 
Walker, R. A., Grigg, M. E., Smith, N. C. and Deplazes, P. 2015 Asexual expansion of Toxoplasma gondii merozoites is distinct from tachyzoites and entails expression of nonoverlapping gene families to attach, invade, and replicate within feline enterocytes. BMC genomics 16(1): 66.

Mangas, T. P., do Couto Rocha, H. P., da Silva Filho, E., Serra-Freire, N. M., and Benigno, R. N. M. 2015. Efficiency of peptide digestion and scarification techniques for detecting Sarcocystis spp. in beef cattle. Coccidia., 2(2): 52-57.

Motamedi, G. R., Dalimi, A., Aghaeipour, K., and Nouri, A. 2010. Ultrastructural and molecular studies on fat and thin macrocysts of Sarcocystis spp. isolated from naturally infected goats. Archives of Razi Institute. 65(2): 91-97.

Mowafy, N. M. 1993 Sarcosporidiosis in rodents. Ph.D. Thesis in Medical Science, Faculty of Medicine, Minia University, Egypt.

Obijiaku, I. N. 2012 Sarcocystis infection in cattle and pigs and its public health implications in Zaria, Nigeria (Doctoral dissertation).

Odening, K. 1998 The present state of species systematics in Sarcocystis lankester, 1882 (Protista, Sporozoa, Coccidia). Systematic Parasitol. 41(3): 209-233.

Odening, K., Stolte, M., and Bockhardt, I. 1996. On the diagnostics of Sarcocystis in cattle: sarcocysts of a species unusual for Bos taurus in a dwarf zebu. Veterinary parasitology. 66(1): 19-24.

Pritt, B., Trainer, T., Simmons-Arnold, L., Evans, M., Dunams, D., and Rosenthal, B. M. 2008. Detection of Sarcocystis parasites in retail beef: a regional survey combining histological and genetic detection methods. Journal of Food Protection®. 71(10): 2144-2147.

Singh, K. P., Agrawal, M. C., and Shah, H. L. 1990. Prevalence of sarcocysts of Sarcocystis capracanis in oesophagus and tail muscles of naturally infected goats. Veterinary parasitology. 36(1): 153-155.

\section{How to cite this article:}

Rasmita Panda, Manchukonda Udaya Kumar, G.S. Sreenivasa Murthy and Reddy, Y.N. 2019. Diagnosis of Bovine Sarcocystosis by Conventional Methods. Int.J.Curr.Microbiol.App.Sci. 8(02): 941-947. doi: https://doi.org/10.20546/ijcmas.2019.802.108 